\title{
Students' Conceptions of Life-Long Learning: an Exploratory Study
}

\author{
Nasser Saleh \\ Integrated Learning Librarian \\ Engineering \& Science Library \\ Queen's University \\ nasser.saleh@queensu.ca
}

\author{
David Strong \\ Professor, NSERC Chair in Design Engineering \\ Faculty of Engineering \& Applied Science \\ Queen's University \\ strongd@appsci.queensu.ca
}

\begin{abstract}
This paper reports on an ongoing study that focuses on students' perceptions of life-long learning and what skills are transferrable after graduation. The pilot qualitative analysis of students' responses focuses on exploring the variations of students' approaches to life-long learning.
\end{abstract}

\section{Introduction}

Life-long learning defined by the CEAB accreditation criteria and procedures [1] as being the learners' ability to identify and to address their own educational needs in a changing world, sufficiently to maintain their competence and contribute to the advancement of knowledge.

The inclusion of life-long learning as a graduating attribute for engineering programs is important as engineering graduates should be equipped with these competencies and skills and know how to develop these skill sets. While there are many definitions for life-long learning and the needed skills that may include: problem solving, critical thinking, and reasoning skills; practical ingenuity; information and technology literacy; self-management skills in completing tasks on time, communication, collaboration, and leadership skills; language skills beyond first language; understanding of professional and ethical responsibilities; understanding of importance of lifelong learning and ongoing professional and personal development [2].

\section{Method}

To get more insights on students' experience in the engineering program and specifically in their senior design course; students near the end of their program were asked to think about life-long learning and what does it mean to them. During a workshop, students worked within their project groups to think-aloud about these skills to answer relevant questions pertaining to lifelong learning. Students were introduced to the definition of life-long learning from the CEAB accreditation criteria and were asked to answer the following questions:

1. What does life-long learning mean to you?

2. What are the life-long learning skills that are needed for an engineering graduate?

3. What are the life-long skills that you have learned in this course?

4. How can you make the LL skills transferable into your future professional life, i.e. what's your strategy?

Each group compiled their answers and one student from each group presented the findings to the class. Students' conceptions showed levels of similarity but also levels of differences as well that required us to undergo further investigation.

Students' responses were collected after the workshop in electronic format and were categorized using NVIVO 8, a qualitative data analysis software to identify the major categories of students' responses. This paper will only report on the first question that we have about what do students think of life-long learning and what are the required skills they are aware of to be essential for life-long learning. The rich and contextual data gathered from the workshop encourages us to make a further analysis of the responses that will be reported in another paper to contribute into the identification of issues less mentioned in the literature.

The study adopts a phenomenographic approach consistent with the focus on describing variations in conceptions of learning. Phenomenography is a research approach which seeks to describe the qualitatively different ways in which people understand a phenomenon [3]. Phenomenography is 
based on the idea that a particular phenomenon can be experienced in a limited number of different ways. A way of experiencing a phenomenon corresponds to a particular 'structure of awareness'. A particular structure of awareness is made up of a number of aspects of a phenomenon, simultaneously present and related in a particular way.

The data analysis will be completed according to the principles for phenomenographic analysis outlined by Marton and Booth [4]. In analyzing the transcripts of the workshop, the distinctions between individuals will be initially ignored, and the separate transcripts will be combined and treated as whole, although the statements in the resultant pool of statements were coded to identify the group transcript each statement originated from. The first stage of the analysis was to identify which statements were relevant to the analysis on the basis that they expressed a way of experiencing how students perceive life-long learning.

The second stage will identify an initial draft set of categories. This will involve the sorting of statements from the transcripts by identifying and grouping the expressed ways of experiencing lifelong learning and its required skills. The sorting will be achieved by focusing on similarities and differences in the meanings expressed, and grouping statements expressing similar meanings.

\section{Discussion}

The preliminary analysis of students' responses showed that students identified many skills that they perceive as life-long learning related skills that include both of professional generic skills and transferable skills that must be transferred from their education to their future professional practice.

Students identified many skills as life-long learning skills such as: communication skills, information searching and organization, critical thinking, problem solving, emotional intelligence, creativity, ability to work in teams, research skills, use of information technology. The definition of lifelong learning for student was found to have many variations such as:

"Lifelong learning entails self identification of core-competencies, deficiencies within competency, and developing effective and meaningful strategies to address these deficiencies”. [Respondent A1].
"Never be content with your knowledge: always room for improvement” [Respondent G1].

“An engineering graduate needs to be aware of innovations within one's own field and industry. Lifelong learning for an engineer incorporates advancement in technology, trends in industry, society, standards and culture” [Respondent B2].

Students' perceptions about the limitation of their own knowledge were also reflected in many responses such as:

"Look for more worn-out ring: ask them questions” [Respondent J2].

"Competency awareness: know your limitations” [Respondent k2].

The preliminary analysis of data suggested us to go further into creating two major categories of students' responses based on what skills are considered to be generic engineering skill and what are considered to be transferrable ones.

\section{Conclusion}

The current study is important to learn more about students' experience at the end of their engineering program and to help us to identify what are the skill sets that can be considered as life-long learning skills as a graduating attributes of engineering graduates. The identification of these skills can help educators to focus on these skills along with their characteristics that can be: conceptual skills, contextual skills, and if these skills are taught to be transferrable. The full analysis of students' responses is still in progress.

\section{References}

[1] . Engineers Canada, Canadian Engineering Accreditation Board, Accreditation Criteria and Procedures. Canadian Council of Professional Engineers, 2009.

[2] P. J. Hager and S. S. Holland, Graduate Attributes, Learning and Employability, Springer, Dordrecht, 2006.

[3] J. Bowden and E. Walsh Phenomenography. RMIT , Melbourne, 2000

[4] F. Marton and S. Booth, Learning and Awareness, Lawrence Erlbaum , Mahwah, NJ, 1997 